\title{
Literaturbericht
}

Marcel Lewandowsky

\section{Eine rechtspopulistische Protestpartei? Die AfD in der öffentlichen und politikwissenschaftlichen Debatte}

\section{Einleitung}

Mit der Alternative für Deutschland (AfD) trat Anfang des Jahres 2013 eine neue Partei auf den Plan, die aus dem Stand heraus eine lebhafte Debatte in der politischen und wissenschaftlichen Öffentlichkeit provozierte. Schon unmittelbar nach ihrer Gründung sah sich die Partei Vorwürfen ausgesetzt, den rechten Rand des bundesdeutschen Parteiensystems abzudecken gar selbst rechtspopulistisch (Arzheimer 2015: 2) beziehungsweise rechtsradikal zu sein (Lachmann 2013). Angesichts des frühen Stadiums ihrer Entwicklung wurde dies allerdings weniger als Befund formuliert, sondern stand als Frage im Raum (bspw. Sieber 2013). Es schien, als wisse man nicht recht, wie diese Partei einzuordnen sei, und das durchaus mit gutem Grund. Zum einen pflegte sie aufgrund der sie damals prägenden Persönlichkeiten (insb. Bernd Lucke, Joachim Starbatty und Alexander Gauland) den Habitus der Professorenpartei, die ihre gegen die gemeinsame europäische Währung gerichteten Positionen mit ökonomischer Vernunft begründete (bspw. AfD 2014). Zum anderen kritisierte die Partei von Beginn an nicht allein den Euro, sondern rückte die Bundesregierung und die entscheidenden Institutionen als gewissermaßen "Schuldige“ ins Blickfeld, während sie sich gleichzeitig von extremistischen Positionen distanzierte (Lewandowsky 2014 a: 5). Von Beginn an pflegt die AfD eine Anti-Establishment-Attitüde, mit der sie sich als „Außenseiter" zu stilisieren versucht und von den etablierten Parteien abgrenzt. Sie weist durchaus Züge auf, die von (rechts)populistischen Parteien bekannt sind. Allerdings kann ein solcher educated guess auf Grundlage der medialen Präsenz der Partei und des Diskurses um ihren Charakter kaum befriedigen, wenn man die Frage nach ihrer Einordnung beantworten will (Lewandowsky 2014 b: 20). Klar ist, dass die AfD eine „Partei im Werden“ war und ist und auch ihre derzeitige 
wissenschaftliche Einordnung daher auf Momentaufnahmen basieren muss. Bislang kann die Forschung deshalb nur Ergebnisse von vorläufigem Bestand anbieten. Gleichwohl scheint der Habitus der AfD gegen die „Altparteien“ und das politische Establishment in Berlin und Brüssel die Frage nach dem Populismus, ihre gesellschaftspolitischen Positionen die des Rechtspopulismus immerhin soweit zu induzieren, dass sich jüngere Arbeiten mit der Frage nach ebendieser Einordnung befassen. ${ }^{1}$

Die Entwicklung der AfD im Nachgang der Bundestags- und der Europawahlen spricht in der Tat dafür, dass sich der Eindruck einer rechtspopulistischen fünften oder sechsten Kraft - unter der Voraussetzung, dass die FDP wieder in den Bundestag zurückkehrt ${ }^{2}$ - im deutschen Parteiensystem bestätigen könnte. Während die Ausrichtung der Partei in den ersten zwölf Monaten ihres Bestehens nicht zuletzt aufgrund ihrer thematischen Fokussierung unstrittig als euroskeptisch galt, die Verortung innerhalb der Familie rechtspopulistischer Parteien aber keineswegs eindeutig ausfallen konnte, machte sich Anfang 2014 eine Kursveränderung innerhalb der AfD bemerkbar, die die bislang nicht prominent platzierten gesellschaftspolitischen Positionen ins Blickfeld rückte. Sichtbar wurde die Erweiterung der Euro-Agenda um kulturelle und familienpolitische Themen auf dem Aschaffenburger Parteitag im Januar 2014, wo sich abzeichnete, dass die Partei „,zu einer rechtskonservativen, gesellschaftskritischen Kraft“ werden sollte, „die gegen alles Mögliche ist - eben auch gegen allerlei Dinge in Europa“ (Amann 2014; siehe auch Ankenbrand 2014).

Dass sich die Forschungsdiskussion nicht zuletzt darum dreht, ob die AfD als rechtspopulistisch bezeichnet werden kann, ist womöglich auch der Tatsache geschuldet, dass dieser Forschungszweig zwar in der Bundesrepublik eine gewisse Popularität besitzt ${ }^{3}$ und Rechtspopulisten in anderen europäischen Staaten zu einer festen Größe in den Parteiensystemen geworden sind, es aber bislang, gemessen am Einzug ins Parlament, an einer auf Bundesebene erfolgreichen rechtspopulistischen Partei fehlt. Bereits der Beinahe-Erfolg der AfD bei der Bundestagswahl 2013, wo sie 4,7 Prozent der Zweitstimmen erhielt, und der - bei nahezu gleichbleibender absoluter Stimmenzahl - reelle Erfolg der Partei bei der darauffolgenden Europawahl (7,1 Prozent) zog einige Arbeiten nach sich, die direkt oder mittelbar danach fragten, ob es sich bei der AfD nun „endlich“ um die

1 Vergleiche hierzu Arzheimer 2015; Bebnowski 2015; Berbuir/Lewandowsky/Siri 2015; Franzmann 2014; Nestler/Rohgalf 2014.

2 Für eine aktuelle Studie zu diesem Thema siehe Patton 2015.

3 Siehe statt vieler die Arbeiten von Pfahl-Traughber 1994; Decker 2004, 2006 b; Häusler 2008; Hartleb 2004; Werz 2003. 
rechtspopulistische Partei handle, die den „Sonderstatus“ der Bundesrepublik in diesem Sinne aufhebt. ${ }^{4}$

\section{Zum Begriff des Rechtspopulismus}

Will man verstehen, wieso sich die Kategorisierung der AfD mit Blick auf den Rechtspopulismus anbietet, aber zugleich an gewisse Grenzen stößt, kommt man nicht umhin, den Begriff selbst zumindest zu skizzieren. Das kann freilich nur in einem kurzen Abriss geschehen; die Literatur zu diesem Thema füllt inzwischen ganze Regale. Grundsätzlich hat Margaret Canovan (1981) mit ihrer Studie womöglich das Initial einer originären Populismusforschung gesetzt. Ein grundlegendes Problem besteht darin, dass der Begriff des Rechtspopulismus selbst lange Zeit umstritten war und sich viele Arbeiten damit befassten, wie denn nun die in den 1980er Jahren aufkommende neue Parteienfamilie in Europa zu bezeichnen sei - den Parteien wohnt etwas Gemeinsames inne; das ideologische bzw. programmatische Erscheinungsbild variiert aber je nach nationalem Kontext stark (Mudde 1996). Nicht ohne Grund identifiziert Cas Mudde (2007) in seiner Monografie 22 Begriffe, die im Wesentlichen denselben Parteientypus beschreiben (siehe auch Decker/Lewandowsky 2012: 270).

Mudde kann als jener Autor gelten, der in der jüngeren internationalen Populismusforschung bedeutend dazu beigetragen hat, eine Minimaldefinition und damit eine konzeptionelle Grundlage des Rechtspopulismus zu entwickeln. Die Basis legte er in einer Verdichtung des Populismus-Begriffes - noch ohne politisches Attribut -, in welcher er diesen als Antagonismus zwischen einem als homogen gedachten Volk und einer dessen politischen Willen entgegen stehenden politischen Elite interpretiert (Mudde 2004). Der Gegensatz des Wir-gegen-Oben ist also konstituierendes konzeptionelles Merkmal des Populismus. Gleichwohl bemerkt Decker (2004: 29), dass die meisten Parteien, die in der Realität als populistisch gelten können, zugleich rechtspopulistisch sind. Rechtspopulismus wird demnach durch eine zweite Abgrenzung definiert, nämlich gegen jene, die als dem Volk nicht zugehörig gedacht werden. Gerade hier allerdings stellt sich das Problem der konzeptionellen Fassung, da die ,äußeren Feinde“ in den Parteien und Ideologien ganz unterschiedlich sein können (Taggart 2000). So teilte beispielsweise die niederländische Lijst Pim Fortuyn die Islamfeindlichkeit mit anderen Parteien dieses Spektrums; Fortuyn warf jedoch als charismatische Führungspersönlichkeit der LPF seinen hedonistischen Lebensstil und seine Homose-

4 So etwa Arzheimer 2015; Berbuir/Lewandowsky/Siri 2015; Franzmann 2014; Lewandowsky 2014 a.). 
xualität in die Waagschale seiner medialen Inszenierung, was bei Parteien wie dem französischen Front National oder der österreichischen FPÖ nicht denkbar wäre. „Rechts“ an dieser Spielart des Populismus ist nach Mudde (2007: 18 ff.) der Nativismus, also die Proklamation von Vorrechten vermeintlich natürlich-einheimischer Gruppen gegenüber ethnischen, religiösen und anderen Minderheiten, die dem populistischen Volksbegriff als „fremd“" gegenüber gestellt werden. ${ }^{5}$ Ist der Populismus also im Kern volkszentriert und gegen das Establishment gerichtet, so wendet sich der Rechtspopulismus zugleich gegen äußere Feinde. Aus dieser ideologischen Grundhaltung erwachsen sowohl organisationale als auch stilistische Merkmale populistischer Parteien (jüngst Rooduijn 2014): Das Selbstverständnis von Populisten, nicht Partei im klassischen Sinne, sondern „Bewegung“ zu sein und sich gegen die Etablierten abzugrenzen, lässt sie klassisch als auf eine charismatische Führungspersönlichkeit zugeschnittene Organisation mit schwacher Binnenstruktur auftreten (Decker 2006 a: $17 \mathrm{f}$.). Jüngere Bewegungen, wie etwa die US-amerikanische Tea Party, deuten jedoch darauf hin, dass dieses Modell ein Stück weit überwunden werden könnte (Mudde/Kaltwasser 2014). Das Varieté populistischer Stilmittel gruppiert sich um einige zentrale Elemente (Decker 2004: 35 f.): So neigen Populisten zu vereinfachender Sprache, mittels derer sie komplexe politische Fragestellungen auf die Verantwortlichkeit „Schuldiger“ und den politischen Wettbewerb auf das Gegenüber von Freund und Feind reduzieren. Gemein ist ihnen überdies der vermeintliche Tabubruch, mit dem sie vorgeben, die ersten zu sein, die - im Gegensatz zum politischen/mediale Mainstream - brisante Themen anzusprechen wagen. Tatsächlich liegt der eigentliche Tabubruch jedoch in der Art und Weise, wie die Themen verhandelt werden (Lewandowsky/Siri 2011): Rechtspopulisten adaptieren häufig biologistische Sprachelemente, Gewaltmetaphorik und adressieren gezielt diffuse Ängste (Decker 2004: 36).

Es nimmt angesichts des Anti-Establishment-Gestus der AfD also nicht wunder, dass sich die Kategorie des (Rechts)Populismus bei der Betrachtung der Partei geradezu aufdrängt. Immerhin geriert sie sich als Protestbewegung gegen Euro, EU und Bundesregierung und sieht sich immer wieder dem Vorwurf ausgesetzt, auch ausländerfeindliche und homophobe Ressentiments zu bedienen. Wie allerdings stellt sich dies in der politikwissenschaftlichen Debatte dar?

5 Van Spanje (2011) hat die fehlende Unterscheidung zwischen rechtsradikalen (far right) und Antiimmigration-Parteien mit dem empirischen Befund kritisiert, dass das Immigrations-Issue und die rechte politische Ausrichtung nicht in kausaler Beziehung zueinander stünden. Allerdings kann dem entgegnet werden, dass Nativismus bzw. Xenophobie für rechtspopulistische Parteien eine notwendige Bedingung darstellen. 


\section{Die Debatte um die Einordnung der AfD}

Eines vorweg: Eine einhellige Ansicht zur AfD findet sich in der Literatur bislang nicht; sie wird nicht einheitlich und eindeutig der rechtspopulistischen Parteienfamilie zugeordnet - allerdings kann sich dies ändern, wenn der nationalkonservative Flügel dauerhaft die Oberhand gewinnen sollte. Im sich gerade entwickelnden Schrifttum lassen sich bislang zwei Stränge unterscheiden. Auf der einen Seite lässt sich eine strenger empirische Ausrichtung ausmachen. Sie hält sich an die Anforderungen qualitativer und quantitativer Datenauswertung; insofern verwundert es nicht, dass sie aufgrund der Datengrundlage (Programme, Pressemeldungen, mediale und Online-Kommunikation) mit einer klaren Typisierung der AfD zurückhaltend sein muss. So verweist etwa Simon Franzmann (2014) auf den nach wie vor nicht abgeschlossenen Gründungs- und Findungsprozess der Partei und kommt mit Blick auf die Programmatik zu dem Schluss, dass vor allem aufgrund ihrer Anti-Establishment-Rhetorik ,thematische und stilistische Ähnlichkeiten der AfD mit rechtspopulistischen Parteien in Europa festgestellt werden [können], aber keine wirkliche Übereinstimmung“ (ebd.: 122). Auch Kai Arzheimer (2015) argumentiert, dass der Partei radikale Positionen zumindest in ihrer offiziellen Programmatik auf Bundesebene weitestgehend fehlen - jene sind es aber, die den rechtspopulistischen Parteientypus in Westeuropa kennzeichnen. ${ }^{6}$ Während Rüdiger Schmitt-Beck im Vorfeld der Bundestagswahl 2013 ebenfalls zu einem zurückhaltenden Schluss mit Blick auf den Rechtspopulismus kommt, erkennt er bereits, „dass es in der Partei Versuche gibt, sie deutlicher in diese Richtung zu positionieren“ (Schmitt-Beck 2014: 112).

Marcel Lewandowsky (2014b) kennzeichnet die AfD im Vergleich mit der Pro-Bewegung als populistisch, spart das ideologische Attribut jedoch aus. Der Vergleich der Online-Kommunikation der AfD mit jener der Bürgerbewegung pro Deutschland vor der Bundestagswahl 2013 zeigt, dass die AfD auch bei Facebook und Twitter vor allem die Euro-Politik der Bundesregierung angreift, $\mathrm{Zu}-$ wanderung bzw. der Islam als Mobilisierungsthema des Rechtspopulismus jedoch keine Rolle spielen. Dieses Bild bestätigt sich, wenn man diese Diskursanalyse auf die mediale Kommunikation mittels Plakaten und Reden ausweitet (Berbuir/ Lewandowsky/Siri 2015). Die AfD geriert sich gewissermaßen ,auf allen Kanälen" als Anti-Establishment-Partei, vermied aber lange Zeit offiziell islamophobe

6 Populistische Parteien in Mittel- und Osteuropa unterscheiden sich hinsichtlich ihrer ideologischen Form und ihrer sozialen bzw. historischen Voraussetzungen zum Teil wesentlich von westeuropäischen Vertretern. Siehe hierzu statt vieler Bachmann (2006); Frölich-Steffen (2008); Deegan-Krause (2012); Hanley (2012). 
und ausländerfeindliche Aussagen. Diese finden sich gewissermaßen subkutan (ebd.; vgl. Bebnowski 2015: 15) beziehungsweise bei Akteuren der ,zweiten Reihe", etwa in Aussagen des Publizisten und brandenburgischen Landes- und Fraktionsvorsitzenden Alexander Gauland, der sich schon früh nicht nur gegen den Euro, sondern auch gegen „Gender-Wahn“ und die multikulturelle Gesellschaft positionierte (Gauland 2013). Arzheimer (2015) kann ebenfalls auf Grundlage eines Mixed-Methods-Designs zeigen, dass die AfD am rechten Rand des Parteienspektrums der Bundesrepublik verortet werden kann. Hierzu generiert er aus dem AfD-Europawahlprogramm 2014 quantitative und qualitative Daten, die durch Daten der Parteikommunikation in den sozialen Netzwerken ergänzt werden. Gleichzeitig kommt Arzheimer (ebd.: 17) zu dem Schluss, dass die AfD auf Grundlage ihrer inhaltlichen Positionierung eben nicht als „radikal“ bezeichnet werden kann. Sein vorsichtiger Befund kann als stellvertretend für diese Richtung der AfD-Forschung angesehen werden.

Diesem Strang stehen normativ angeleitete Arbeiten gegenüber, die die AfD bereits früh, also unmittelbar nach ihrem Erscheinen auf der politischen Bühne der Bundesrepublik, in den Kontext der Neuen Rechten einordneten. $\mathrm{Zu}$ nennen sind hier vor allem die Arbeiten von Alexander Häusler (2013, 2014; Häusler/ Roeser 2015). Häusler kam bereits in seiner ersten Studie, die insgesamt auch eine der ersten Arbeiten zur AfD darstellt, zu dem Befund, dass der Partei guten Gewissens eine „durch viele Aussagen aus deren Parteimilieu belegbare Tendenz zu rechtspopulistischer Ausrichtung" (ebd.: 92) unterstellt werden könne. In einer für den Deutschen Gewerkschaftsbund im März 2014 erstellten Analyse präzisiert er: „Die AfD kann hinsichtlich ihrer in dieser Studie aufgezeigten politischen Positionierungen als eine Partei rechts der CDU/CSU und der FDP stehend eingeordnet werden, die sowohl nationalliberale und nationalkonservative wie zudem auch rechtspopulistische Tendenzen aufweist.“ (Häusler 2014: 113)

David Bebnowski (2015) nimmt in seiner jüngst erschienenen Monografie vor allem die einzelnen Akteure innerhalb der Partei in den Blick. Angeschlossen an eine kritische Diskussion des Rechtspopulismus-Begriffs (ebd.: $13 \mathrm{ff}$.) stellt auch Bebnowski heraus, dass die AfD sich in ihrer Rhetorik von anderen Exponenten dieser Parteienfamilie unterscheidet: „Interessant ist im Fall der AfD jedoch, dass sie hierbei in weiten Teilen auch ohne die in der Einleitung angesprochenen Ausfälligkeiten auskommt. Der Populismus der AfD funktioniert häufig subtiler.“ (ebd.: 15) Er stellt heraus, dass sich die spezifische Form des Populismus der AfD in der „Logik ökonomischer Konkurrenz und den Leitbildern des Neoliberalismus [zeigt]. Bereits durch ein Bemühen der Wettbewerbslogik lassen sich Ängste schüren und sind Abwertungen des Anderen zu erreichen.“ 
Ein Unterschied zu jenen Arbeiten, die eine strengere Empirie verfolgen, liegt in der hermeneutischen Grundausrichtung, die darauf angelegt ist, die AfD auf Grundlage einer umfassenden Materialauswahl in ihrer aktuellen Gestalt zu verstehen. Hier schließt die Frage nach den organisationalen Merkmalen des Populismus an (Mudde/Kaltwasser 2014; Rooduijn 2014). Im Gegensatz zu klassischen Vertretern dieser Spielart, wie der DVU unter Gerhard Frey oder der Schill-Partei (Decker/Hartleb 2006: 192), ist die AfD alles andere als eine „Führerpartei“. Einerseits wird ihr Vorsitzender Bernd Lucke immer wieder von innerparteilichen Konkurrenten herausgefordert und dessen Politik, etwa in der Frage der Organisationsstruktur, massiv infrage gestellt (Werner 2015; Blome 2015). Auch das Auftreten als Experten- bzw. „Professorenpartei“ (Giesa 2013; Sieber 2013) - die AfD hält sich als einzige Partei einen „Wissenschaftlichen Beirat“ auf der Bundesebene - spricht für eine grundlegend andere organisationale Selbstbeschreibung als etwa des Front National unter Jean-Marie Le Pen, der als ,Junge aus der Bretagne“ mit dem „einfachen Volk“ fraternisierte. Andererseits haben Mudde und Kaltwasser (2014) jüngst herausgearbeitet, dass sich gerade jüngere Bewegungen durch das Fehlen charismatischer Führungspersönlichkeiten auszeichnen (leaderless populism).

Mit Blick auf den Bewegungscharakter ist es aber gerade sinnvoll, sich den die Partei tragenden Personennetzwerken sowie der Beobachtung der gesellschaftlichen Vernetzung über die reine Betrachtung des Elektorates hinaus zuzuwenden. Dies wiederum stellt bislang eher ein Alleinstellungsmerkmal dieser Forschungsrichtung dar (Bebnowski 2015; Häusler 2013, 2014). Dazu zählt auch die zumindest temporäre punktuelle Kooperation der AfD mit der Pegida-Bewegung, die auf strategische Anknüpfungspunkte der Partei mit konservativen Gruppierungen, möglicherweise sogar der radikalen Rechten hindeuten könnte (Geiges/Marg/ Walter 2015: $152 \mathrm{ff}$.).

Will man zwischen den eher normativen und den eher empirisch angeleiteten Arbeiten eine Schnittmenge hinsichtlich ihrer Ergebnisse ziehen, so liegt diese in dem Schluss, dass die AfD relativ eindeutig als populistisch bezeichnet werden kann. ${ }^{7}$ In der Frage nach dem „rechten Grad“ dieser Orientierung können wir in der Literatur so etwas wie ein Spannungsverhältnis zwischen apriorischer Setzung und empirischer Vorsicht erkennen: erscheint die Partei in normativ angeleiteten Arbeiten von vornherein als Exponentin der Neuen Rechten, so können durch die Datenfixierung stärker empirischer Arbeiten nur Ergebnisse unter Vorbehalt produziert werden. Noch fehlt der Partei allerdings, im Gegensatz zu etablierten Ver-

\footnotetext{
7 Abweichend Arzheimer (2015: 546, 551).
} 
tretern der rechtspopulistischen Parteienfamilie wie der FPÖ, dem Front National oder der Partij voor de Vrijheid in den Niederlanden, die fixierte xenophobe Programmatik. Bislang changiert die AfD ,zwischen marktliberalen, auf ökonomische Expertise rekurrierenden Positionen und einer nationalkonservativen Rhetorik, die zielsicher tiefsitzende Stereotype und Ressentiments bedient" (Nestler/ Rohgalf 2014: 411; Hervorhebungen durch den Autor).

\section{Die AfD als neuer Akteur im Parteiensystem}

Das Auftauchen einer relativ erfolgreichen populistischen Partei erzeugt in der politischen Öffentlichkeit der Bundesrepublik einen besonderen Nachhall. Die Erfahrung des Dritten Reiches, die den Diskurs bis heute prägt, bringt es mit sich, dass Parteien rechts von CDU und CSU rasch in den Verdacht der ideologischen Nähe zum Nationalsozialismus geraten (Decker 2005). Im Gegensatz zu anderen europäischen Staaten, etwa den Niederlanden oder Frankreich, wo Geert Wilders bzw. Marine Le Pen zur Prime Time an politischen Talkformaten teilnehmen, stehen die seltenen Auftritte rechter Politiker unter dem Damoklesschwert des Nazismusverdachts.

Dieser Abwehrreflex, der nicht immer als durchdachte Strategie zu verstehen ist, beruht auf der Perzeption, dass eine Partei rechts von CDU und CSU zugleich auch radikal oder gar extremistisch sein muss. In der Tat entsprachen die zuletzt auf Landesebene (NPD, zuvor auch DVU) und in einigen Kommunen (Pro-Bewegung) erfolgreichen Parteien diesem Bild (Lewandowsky 2012: 398 ff.). Als die AfD auf der Bühne auftauchte, versuchten die etablierten Parteien, diesen Weg erneut zu beschreiten. Die AfD tat ihnen jedoch lange Zeit nicht den Gefallen, das Stigma des Rechtsradikalismus ohne weiteres zu erfüllen (ders. 2014 b). Die Stigmatisierungsstrategie beruht zum einen auf der zumindest latenten Systemfeindlichkeit solcher Parteien, zum anderen auf deren offen ausgespielter Xenophobie. Bis zur Europawahl 2014 bemühte sich die AfD, ein solches Bild in der Öffentlichkeit zu vermeiden. Sie gerierte sich als eurokritische Anti-Establishment-Partei. Dass diese Begriffe zusammenhängen, zeigt eine von Morten Pieper, Stefan Haußner und Michael Kaeding (2015) erstellte qualitative Analyse des Euroskeptizismus. Sie können zeigen, dass die Europakritik von der AfD nicht zuletzt innenpolitisch gewendet wird (ebd.: $158 \mathrm{f}$.). Bei der Europawahl wurde sie überdies nicht allein aus Protest, sondern aufgrund ihrer europapolitischen Thematik und Positionierung in diesem Feld gewählt (Wagner/Lewandowsky/Giebler 2015). Sie ist damit nicht allein Protestpartei in dem Sinne, dass sie als Projektionsfläche gegen die etablierten Parteien dient, sondern hatte bis dato ein attraktives themati- 
sches Portfolio anzubieten - allein schon deshalb dürfte die Stigmatisierungsstrategie wenig aussichtsreich sein. Tatsächlich zeigen Daten, dass diese Strategie durch die AfD verfing. Sie wurde auf der Wählerseite als nur unwesentlich weiter rechts stehend als die CSU wahrgenommen (ebd.: 145).

Erreichte die Partei beim europäischen Urnengang am 25. Mai 2014 einen vorläufigen Höhepunkt - zumindest, was den prozentualen Stimmenanteil betrifft -, so konnte sie im Herbst desselben Jahres an diesen Erfolg anknüpfen. Bei den Landtagswahlen in Brandenburg, Sachsen und Thüringen konnte die AfD hohe Stimmergebnisse erzielen. Die Programmatik der Landesverbände war allerdings wenig überraschend kaum europapolitisch geprägt, sondern griff kulturelle und integrationspolitische Themen auf. So plädierte der Landesverband Brandenburg in seinem Wahlprogramm für eine Förderung der ,kulturellen Identität“ des Landes (AfD Brandenburg 2014: 7 f.) und forderte zugleich eine höhere Integrationsbereitschaft von Zuwanderern (ebd.: 8). Der Landesverband Thüringen forderte die Einführung von Volksentscheiden zu diesem Thema; überdies müsse die „Zuwanderung in die deutschen Sozialsysteme [...] unbedingt unterbunden werden" (AfD Thüringen 2014: 8). Die AfD in Sachsen blies in ein ähnliches Horn; sie kontrastierte die Kosten der Zuwanderung ,mit einem Ausbau der Familienförderung und mit Qualifikationsangeboten für deutsche Arbeitslose“ (AfD Sachsen 2014: 17).

Die Erfolge bei den Landtagswahlen dürften indes die strategische Ausgangslage der AfD nicht unbedingt verbessert haben. Die günstige Basis zum Zeitpunkt der Europawahl rührte aus der Kombination von moderat-rechter Verortung bei gleichzeitig stark euroskeptischer Positionierung. Sie war daher abhängig von der Salienz des europapolitischen Themas (Wagner/Lewandowsky/Giebler 2015). Es nimmt nicht wunder, dass die Partei auf der Landesebene andere Themen suchte und damit auch erfolgreich war. Zugleich rückte dadurch aber eine innerparteiliche Strömung ins Blickfeld, die vor allem auf gesellschaftspolitische Themen setzt. In der Folge ist innerhalb der AfD ein von den Medien dankbar aufgenommener Richtungsstreit entbrannt, der im Wesentlichen zwischen den als - im Vergleich -eher moderat geltenden, wirtschaftsliberal-euroskeptischen Kräften um Bernd Lucke und dem inzwischen aus dem Bundesvorstand zurückgetretenen Hans-Olaf Henkel sowie einem nationalkonservativen Flügel um Frauke Petry und Alexander Gauland schwelt. Das illustriert das der AfD zugrunde liegende Dilemma: Auf der einen Seite würde ein gemäßigter, euroskeptischer Kurs die Partei ohne Alleinstellungsmerkmal zu nah an die Wettbewerbsposition der Union heranführen; andererseits droht eine Partei, die sich gegen die Vielfalt von Lebens- bzw. Partnerschaftsmodellen und für eine härtere Integrationspolitik aus- 
spricht, der politischen Stigmatisierung zum Opfer zu fallen. Hinzu kommt, dass die Partei gerade in den Monaten nach den Landtagswahlen vor allem durch interne Richtungskämpfe von sich reden machte, die sich sowohl am Kurs der Partei als auch an der Führungsstruktur festmachten (bspw. Soldt 2014).

Es ist noch nicht abzusehen, ob mit Blick auf die Nachfrageseite des Parteienwettbewerbs ein eher moderater oder radikaler Kurs für die AfD erfolgversprechend wäre. Bislang sprechen die Befunde keine eindeutige Sprache. Betrachtet man die Wählerwanderung der AfD bei allen Wahlen seit der Bundestagswahl 2013, so gewann sie, neben dem Nichtwählerlager, vor allem aus den Reihen der Union, der FDP und der Linken (Zeit Online 2013). Bei den Landtagswahlen in Brandenburg und Thüringen nahm die Union jeweils den ersten, die Linke den zweiten Platz in der Wählerwanderungsbilanz ein, während sie bei der Europawahl mit Abstand die meisten Wähler von der Union gewann - fast dreimal so viele wie von den Sozialdemokraten (Rietzschel 2014). Das spricht zunächst dafür, dass die AfD protestorientierte Wähler aus nahezu allen Lagern für sich gewinnen kann, was wiederum die SPD koalitionspolitisch vor Probleme stellen könnte (von Lucke 2014). Die AfD ist auch elektoral insoweit „populistisch“, als die lagerübergreifende Wanderung für eine gemeinsame Protestorientierung der Wähler spricht (Korte/Leggewie/Lewandowsky 2015). Allerdings ist, abgesehen von der Parteianhängerschaft, die Wählerschaft der AfD relativ homogen, was ihre Skepsis gegenüber Europa und die Ablehnung weiterer Zuwanderung betrifft (Köcher 2014). Eine andere Studie zeigt unter denjenigen Befragten, bei denen die AfD vor der Bundestagswahl hohe Beliebtheitswerte erzielte (mindestens 8 auf einer Skala von 1 bis 10), bei einer leichten Abweichung ein ähnliches Bild. So ist die Ablehnung der EU bzw. des Euro weitaus weniger stark ausgeprägt als ablehnende Haltungen gegenüber dem Adoptionsrecht für homosexuelle Paare oder dem Islam (Berbuir/Lewandowsky/Siri 2015: 172). Auf der Wählerseite scheint die AfD demnach zum einen über ein relativ hohes Potenzial bis in moderat-konservative Gruppen hinein zu verfügen. Andererseits zieht sie Sympathisanten an, die über ein sehr wertkonservatives Weltbild verfügen. Zwischen diesen beiden Polen sieht sich die AfD mit einer Chance auf dauerhafte Etablierung (Köcher 2014), aber auch einem strategischen Dilemma konfrontiert, das zwei Jahre nach der letzten Bundestagswahl bei Weitem noch nicht gelöst ist. 


\section{Fazit und Forschungsperspektiven}

Der schnelle Aufstieg und die damit einhergehende Präsenz der AfD hat die politikwissenschaftliche Forschung rasch auf den Plan gerufen. Ein wenig erscheint es, als habe man geradezu darauf gewartet, mit der AfD nun „endlich“ eine erfolgreiche rechtspopulistische Partei in der Bundesrepublik vorzufinden. Die Forschung oszilliert bislang zwischen der recht eindeutigen Einordnung der AfD in den Kontext der Neuen Rechten und zurückhaltenden Ergebnissen. Dabei stehen diese beiden Forschungsstränge keineswegs im Widerspruch zueinander. Es zeigt sich vielmehr, dass die methodologische Offenheit des normativen Zugangs auch empirische Arbeiten inspirieren kann. Hierzu zählt insbesondere, dass sich ganz offensichtlich zeigt, dass der Charakter der AfD allein durch ihre Programmatik (Franzmann 2014) nicht abgebildet werden kann, weshalb sich Mixed-MethodsDesigns anbieten (Arzheimer 2015; Berbuir/Lewandowsky/Siri 2015). Auch mit Blick auf die Frage, wie Rechtspopulismus messbar gemacht werden kann, wenn er sich eben „subtiler“ äußert (Bebnowski 2015: 15), bietet es sich an, Studien zur AfD stärker querschnittsorientiert aufzubauen beziehungsweise, auch aus forschungspraktischen Gründen, empirische Zugänge jenseits der Programmatik zu entwickeln. Auch elaborierte Ansätze, wie sie vor allem in der englischsprachigen Forschung entwickelt wurden, stoßen dort an ihre Grenzen, wo Populismus/ Rechtspopulismus anhand der offiziellen Programme gemessen wird, also statisch bleiben muss (Rooduijn/Pauwels 2011; Rooduijn/de Lange/van der Brug 2014). Insofern wird sich zeigen, ob aus der Untersuchung der AfD, ihrer Strukturen, ihrer Kommunikation, ihrer internen Dynamik und der sie tragenden Personenkreise auch Inspiration für die weitere Erforschung (rechts)populistischer Parteien erwachsen kann.

Zumindest für den Moment jedoch kann die Frage, ob mit der AfD nun eine rechtspopulistische Partei in der Bundesrepublik Wurzeln schlägt, nicht abschließend beantwortet werden. Hier gilt es zunächst, überhaupt erst die Etablierung der AfD abzuwarten, die mit einem Einzug in den Bundestag steht und fällt. Ansonsten könnte die Partei alsbald Geschichte sein - ein Schicksal, das sie mit den Republikanern und den Piraten gemein hätte. Insofern würde sie in der Tat ein Stück Normalität im deutschen Parteiensystem repräsentieren. 


\section{Literatur}

AfD Brandenburg, 2014: Bodenständig und frei leben. Das Programm der Alternative für Deutschland für die Landtagswahl vom 14. September 2014.

AfD Sachsen, 2014: Wahlprogramm 2014, Langfassung. Auf dem Parteitag am

2. März 2014 in Zwickau beschlossene Fassung.

AfD Thüringen, 2014: Wahlprogramm der Alternative für Deutschland in Thüringen zur Landtagswahl am 14. September 2014.

AfD, 2014: Mut zu Deutschland. Für ein Europa der Vielfalt. Programm der Alternative für Deutschland (AfD) für die Wahl zum Europäischen Parlament am 25. Mai 2014. Beschluss des Bundesparteitags vom 22. März 2014.

Amann, Melanie, 2014: AfD-Parteitag: Der Kampf gegen den Euro war gestern, Spiegel Online, 25.01.14, http://www.spiegel.de/politik/deutschland/afd-parteitag-kurswechsel-der-euro-skeptiker-a-945544.html (Stand: 13.05.15).

Ankenbrand, Hendrik, 2014: Christliche Alternative für Deutschland, Frankfurter Allgemeine Zeitung Online, 10.03.14, http://www.faz.net/aktuell/afd-kritisiert-rechte-von-schwulen-und-muslime-12837646.html (Stand: 13.05.15).

Arzheimer, Kai, 2015: The AfD: Finally a Successful Right-Wing Populist Eurosceptic Party for Germany?, in: West European Politics 38 (3), 535-556.

Bachmann, Klaus, 2006: Populistische Parteien und Bewegungen in Mittelosteuropa, in: Frank Decker (Hrsg.), Populismus. Gefahr für die Demokratie oder nützliches Korrektiv?, Wiesbaden, 216-232.

Bebnowski, David, 2015: Die Alternative für Deutschland. Aufstieg und gesellschaftliche Repräsentanz einer rechten populistischen Partei, Wiesbaden.

Berbuir, Nicole/Lewandowsky, Marcel/Siri, Jasmin, 2015: The AfD and its Sympathisers: Finally a Right-Wing Populist Movement in Germany?, in: German Politics 24 (2), 154-178.

Blome, Nikolaus, 2015: Krise der AfD: Ende einer Nicht-Partei, Spiegel Online, 12.05.15, http://www.spiegel.de/politik/deutschland/afd-nikolaus-blome-ueber-das-ende-einer-nicht-partei-a-1033395.html (Stand: 13.05.15).

Canovan, Margaret, 1981: Populism, London.

Decker, Frank (Hrsg.), 2006 b: Populismus. Gefahr für die Demokratie oder nützliches Korrektiv?, Wiesbaden.

Decker, Frank, 2004: Der neue Rechtspopulismus, Opladen.

Decker, Frank, 2005: In Hitlers Schatten, Die Zeit, 26.02.05, 6.

Decker, Frank, 2006 a: Die populistische Herausforderung. Theoretische und ländervergleichende Perspektiven, in: Ders. (Hrsg.), Populismus. Gefahr für die Demokratie oder nützliches Korrektiv?, Wiesbaden, 9-32. 
Decker, Frank/Hartleb, Florian, 2006: Populismus auf schwierigem Terrain. Die rechten und linken Herausfordererparteien in der Bundesrepublik, in: Frank Decker (Hrsg.), Populismus. Gefahr für die Demokratie oder nützliches Korrektiv?, Wiesbaden, 191-215.

Decker, Frank/Lewandowsky, Marcel, 2012: Die rechtspopulistische Parteienfamilie, in: Uwe Jun/Benjamin Höhne (Hrsg.), Parteienfamilien. Identitätsbestimmend oder nur noch Etikett?, Opladen, 268-281.

Deegan-Krause, Kevin, 2012: Populism, democracy, and nationalism in Slovakia, in: Cas Mudde/Cristóbal Rovira Kaltwasser (Hrsg.), Populism in Europe and the Americas. Threat or Corrective to Democracy?, Cambridge, 182-204.

Franzmann, Simon T., 2014: Die Wahlprogrammatik der AfD in vergleichender Perspektive, in: Mitteilungen des Instituts für Deutsches und Internationales Parteienrecht und Parteienforschung 20, 115-124.

Frölich-Steffen, Susanne, 2008: Populismus im Osten und im Westen als parallele Entwicklungen?, in: Ellen Bos/Dieter Segert (Hrsg.), Osteuropäische Demokratien als Trendsetter? Parteien und Parteiensysteme nach dem Ende des Übergangsjahrzehnts, Opladen/Farmington Hills, 303-321.

Gauland, Alexander, 2013: Brief an die konservativen Parteifreunde, 29. Dezember 2013, http://www.alternativefuer.de/brief-konservative-parteifreunde (Stand: 13.05.15).

Geiges, Lars/Marg, Stine/Walter, Franz, 2015: Pegida. Die schmutzige Seite der Zivilgesellschaft?, Bielefeld.

Giesa, Christoph, 2013: Denkfehler einer Professorenpartei, The European, 27.03.13, http://www.theeuropean.de/christoph-giesa/6673-die-ideen-der-alternative-fuer-deutschland (Stand: 13.05.15).

Hanley, Seán, 2012: The Czech Republicans 1990-1998: a populist outsider in a consolidating democracy, in: Cas Mudde/Cristóbal Rovira Kaltwasser (Hrsg.), Populism in Europe and the Americas. Threat or Corrective to Democracy?, Cambridge, 68-87.

Hartleb, Florian, 2004: Rechts- und Linkspopulismus. Eine Fallstudie anhand von Schill-Partei und PDS, Wiesbaden.

Häusler, Alexander (Hrsg.), 2008: Rechtspopulismus als „Bürgerbewegung“. Kampagnen gegen Islam und Moscheebau und kommunale Gegenstrategien, Wiesbaden.

Häusler, Alexander, 2013: Die „Alternative für Deutschland“ - eine neue rechtspopulistische Partei? Materialien und Deutungen zur vertiefenden Auseinandersetzung, Düsseldorf. 
Häusler, Alexander, 2014: Mut zur Wahrheit? Entstehungskontext, Entwicklung und gesellschaftspolitische Positionen der „Alternative für Deutschland“. Hintergrundinformationen und Analysen, Berlin.

Häusler, Alexander/Roeser, Rainer, 2015: Die rechten ,Mut'-Bürger. Entstehung, Entwicklung, Personal \& Positionen der „Alternative für Deutschland“, Hamburg.

Köcher, Renate, 2014: Die scharfe Kante der AfD, Frankfurter Allgemeine Zeitung, 22.10.14, 8.

Korte, Karl-Rudolf/Leggewie, Claus/Lewandowsky, Marcel, 2015: Die Alternativen der AfD im Klima der Großen Koalition. Eine Partei am Scheideweg zwischen Nationalliberalismus und Rechtspopulismus, in: Blätter für deutsche und internationale Politik 60 (6).

Lachmann, Günther, 2013: Ist die Anti-Euro-Partei rechtsradikal?, Welt Online, 18.03.13, http://www.welt.de/politik/deutschland/article114542461/Ist-dieAnti-Euro-Partei-rechtsradikal.html (Stand: 13.05.15).

Lewandowsky, Marcel, 2012: Rechtspopulismus als Herausforderung für die Demokratie in der Bundesrepublik Deutschland, in: Christian Krell/Tobias Moerschel (Hrsg.), Demokratie in Deutschland. Zustand, Herausforderungen, Perspektiven, Wiesbaden, 389-411.

Lewandowsky, Marcel, 2014 a: Alternative für Deutschland (AfD) - A new actor in the German party system, London.

Lewandowsky, Marcel, 2014 b: Populismus in sozialen Netzwerken: Die Beispiele AfD und pro Deutschland, in: Osnabrücker Beiträge zur Sprachtheorie 86, 19-46.

Lewandowsky, Marcel/Siri, Jasmin, 2011: Warnschüsse aus der Mitte, in: Berliner Republik 3, 87-89.

Mudde, Cas, 1996: The War of Words Defining the Extreme Right, in: West European Politics 19 (2), 225-248.

Mudde, Cas, 2004: The Populist Zeitgeist, in: Government and Opposition 39 (3), 541-563.

Mudde, Cas, 2007: Populist Radical Right Parties in Europe, Cambridge.

Mudde, Cas/Kaltwasser, Cristobál Rovira, 2014: Populism and Political Leadership, in: Rhodes, R. A. W./'t Hart, Paul (Hrsg.), The Oxford Handbook of Political Leadership, Oxford, 376-388.

Nestler, Christian/Rohgalf, Jan, 2014: Eine deutsche Angst - Erfolgreiche Parteien rechts von der Union. Zur AfD und den gegenwärtigen Gelegenheitsstrukturen des Parteienwettbewerbs, in: Zeitschrift für Politik 61 (4), 389-413. 
Patton, David F., 2015: The Prospects of the FDP in Comparative Perspective: Rest in Peace or Totgesagte leben länger?, in: German Politics 24 (2), 179-194.

Pfahl-Traughber, Armin, 1994: Volkes Stimme? Rechtspopulismus in Europa, Bonn.

Pieper, Morten/Haußner, Stefan/Kaeding, Michael, 2015: Die Vermessung des Euroskeptizismus der Alternative für Deutschland (AfD) im Frühjahr 2014, in: Michael Kaeding/Niko Switek (Hrsg.), Die Europawahl 2014. Spitzenkandidaten, Protestparteien, Nichtwähler, Wiesbaden, 149-160.

Rietzschel, Antonie, 2014: Dann geh ich halt zur AfD, Süddeutsche Zeitung Online, 15.09.14, http://www.sueddeutsche.de/politik/waehlerwanderung-inthueringen-und-brandenburg-dann-geh-ich-halt-zur-afd-1.2129805 (Stand: 13.05.15).

Rooduijn, Matthijs, 2014: The Nucleus of Populism. In Search of the Lowest Common Denominator, in: Government and Opposition 49 (4), 573-599.

Rooduijn, Matthijs/de Lange, Sarah Leah/van der Brug, Wouter, 2014: A populist Zeitgeist? Programmatic contagion by populist parties in Western Europe, in: Party Politics 20 (4), 563-575.

Rooduijn, Matthijs/Pauwels, Teun, 2011: Measuring Populism: Comparing Two Methods of Content Analysis, in: West European Politics 34 (6), 1272-1283.

Schmitt-Beck, Rüdiger, 2014: Euro-Kritik, Wirtschaftspessimismus und Einwanderungsskepsis: Hintergründe des Beinah-Wahlerfolges der Alternative für Deutschland (AfD) bei der Bundestagswahl 2013, in: Zeitschrift für Parlamentsfragen 45 (1), 94-112.

Sieber, Roland, 2013: Professorenpartei als rechtspopulistische Sammelbewegung?, Publikative.org, 19.03.13, http://www.publikative.org/2013/03/19/afdprofessorenpartei-als-rechtspopulistische-sammelbewegung (Stand: 25.03.15). Soldt, Rüdiger, 2014: Im munteren Querulantenstadl, Frankfurter Allgemeine Zeitung, 06.10.14, 4.

Taggart, Paul, 2000: Populism, Buckingham.

Taggart, Paul, 2004: Populism and representative politics in contemporary Europe, in: Journal of Political Ideologies 9 (3), 269-288.

van Spanje, Joost, 2011: The Wrong and the Right: A Comparative Analysis of ,Anti-Immigration' and ,Far Right' Parties, in: Government and Opposition 46 (3), 293-320.

von Lucke, Albrecht, 2014: Keine Koalitionen ohne die CDU, taz online, 16.09.14, http://www.taz.de/!146022 (Stand: 13.05.15). 
Wagner, Aiko/Lewandowsky, Marcel/Giebler, Heiko, 2015: Alles neu macht der Mai? Die AfD und die Europawahl 2014, in: Michael Kaeding/Niko Switek (Hrsg.), Die Europawahl 2014. Spitzenkandidaten, Protestparteien, Nichtwähler, Wiesbaden, 137-148.

Werner, Alban, 2015: Vor der Zerreißprobe: Wohin treibt die AfD?, in: Blätter für deutsche und internationale Politik 2, 83-90.

Werz, Nikolaus (Hrsg.), 2003: Populismus. Populisten in Übersee und Europa, Opladen.

Zeit Online, 2013: Die Wählerwanderung im Überblick, 23.09.13, http:// www.zeit.de/politik/deutschland/2013-09/waehlerwanderung-bundestagswahl-2013 (Stand: 13.05.15).

Korrespondenzanschrift:

Dr. Marcel Lewandowsky

Helmut-Schmidt-Universität/Universität der Bundeswehr Hamburg

Institut für Politikwissenschaft

Holstenhofweg 85

22043 Hamburg

E-Mail: lewandowsky@hsu-hh.de 